\title{
Comparison by Simultaneous Measurement of Lens Accommodation and Convergence in 3D Vision and Their Distributions
}

\author{
T. Kojima, Y. Matsuura, M. Miyao, T. Shiomi, and H. Takada
}

\begin{abstract}
In general, disagreement between accommodation and convergence is considered to be a main reason for the visual fatigue and the visually induced motion sickness (VIMS). In this study, we devised a method to simultaneously measure lens accommodation and convergence, and used it to characterize 3D vision. Time courses of these fixation distances and their distributions are compared while they viewed $2 \mathrm{D}$ and $3 \mathrm{D}$ video clips. As a result, the convergence almost agreed with the distance between a subject and a 3D image. Therefore, we succeeded in measuring convergence while the distance of the accommodation focal length shifted.
\end{abstract}

Index Terms -3D movie, motion sickness, accommodation, convergence.

\section{INTRODUCTION}

Recently, the use of 3D images has been spreading rapidly, such as in 3D films and 3D televisions. Despite this increase in $3 \mathrm{D}$ display products and the many studies of stereoscopic vision, the influence of stereoscopic vision on human visual functions remains insufficiently understood [1], [2]. In considering the safety of viewing virtual 3D objects, investigations of the influence of stereoscopic vision on the human body are important. People often report symptoms such as eye fatigue and 3D sickness when viewing 3D images for a prolonged period of time. However, such problems are unreported for so-called natural vision. One of the reasons often given for these symptoms is that lens accommodation and convergence are inconsistent during the viewing of $3 \mathrm{D}$ images.

Accommodation is a reaction that occurs due to differences of refractive power achieved by the action of the musculus ciliaris changing the curvature of the lens and the elasticity of the lens. The result is that an image of the external world is focused on the retina. Convergence is a movement where both eyes rotate in their sockets, functioning to concentrate the eyes on one point in space.

Manuscript received April 26, 2012; revised July 2, 2013. This work was supported in part by the Ministry of Education, Culture, Sports, Science and Technology, Grant-in-Aid for Young Scientists (B) Number 23790658, Grant-in-Aid for Scientific Research (B) Number 24300046, and the Hori Information Science Promotion Foundation.

T. Kojima, M. Miyao, and T. Shiomi are with Graduate School of Information Science, Nagoya University, Furo-cho Chikusa-ku Nagoya, Aichi, 464-8603 Japan (e-mail: mmiyao@is.nagoya-u.ac.jp).

Y. Matsuura is with Headquarters for Innovative Society-Academia Cooperation, University of Fukui, 3-9-1 Bunkyo Fukui-City, Fukui, 910-8507 Japan (e-mail: matuura@u-fukui,ac.jp).

H. Takada is with Graduate School of Engineering, University of Fukui, 3-9-1 Bunkyo Fukui-City, Fukui, 910-8507 Japan (tel.: +81-776-27-8795; fax: +81-776-27-8420; e-mail: takada@u-fukui.ac.jp).
There is a relationship between accommodation and convergence, and this is one factor that enables humans to see one object with both eyes. Convergence occurs when an image is captured differently by each eye (parallax). At the same time, focusing on an object is achieved by accommodation.

The main method of presenting 3D images involves the use of the mechanism of binocular vision, and many improvements have been made in this technology [3], [4]. Much of the literature contends that accommodation is always fixed on the screen where the image is displayed, while convergence intersects at the position of the stereo images. As a result, eye fatigue, 3D sickness, and other symptoms occur [5], [6].

However, we obtained results that indicate an inconsistency between accommodation and convergence does not occur [7]. Even so, viewing inconsistency is still often given a cause of eye symptoms. In a previous study, we could not simultaneously measure accommodation and convergence. In order to resolve this inconsistency, we need to be able to measure accommodation and convergence simultaneously. Therefore, we developed a method to simultaneously measure accommodation and convergence.

A comparative measurement of natural vision is essential in investigating stereoscopic vision.

We performed comparative measurements of $3 \mathrm{D}$ vision, and show the results together.

\section{MATERIAL AND METHODS}

We conducted two experiments. The first experiment was performed in order to confirm whether or not this method of simultaneous measurement could be performed accurately. The second experiment was performed to compare natural and $3 \mathrm{D}$ vision. The devices used in these experiments were an autorefractor/keratometer, WAM-5500 (Grand Seiko Co. Ltd., Hiroshima, Japan) and an eye mark recorder, EMR-9 (NAC Image Technology Inc., Tokyo, Japan). Subjects were given a full explanation of the experiment in advance and consent was obtained. Subjects used their naked eyes or, as needed, wore soft contact lenses, and their refraction was corrected to within \pm 0.25 diopter (a "diopter" is the refractive index of lens and indicates the accommodation power. It is defined as the inverse of meters; for example, 0 stands for infinity, 0.5 stands for $2 \mathrm{~m}, 1$ stands for $1 \mathrm{~m}, 1.5$ stands for $0.67 \mathrm{~m}, 2$ stands for $0.5 \mathrm{~m}$, and 2.5 stands for $0.4 \mathrm{~m}$ ). The WAM-5500 and EMR-9 devices were integrated, and we simultaneously measured the focused distances of accommodation and convergence while the subjects gazed at 2D and 3D images. 
Another experiment was done with ten healthy young males (age: 21-46). As with the previous experiment, the accommodation and convergence of the subjects were measured as they gazed at an object (tennis ball: diameter 7 $\mathrm{cm}$ ) and a 3D video clip presented in front of them in binocular vision. The object and the video clip showed exactly the same motion, moving within a range of $0.6 \mathrm{~m}$ to $1.6 \mathrm{~m}$ in 10 seconds, stopping at positions of $0.6 \mathrm{~m}$ and $1.6 \mathrm{~m}$ for 2 seconds, and moving again in four cycles of about 58 seconds. During this time, the subjects were asked to gaze at these objects, and we measured their accommodation and convergence. This measurement of the real object and the 3D video clip was taken four times. The illuminance of the environment and the brightness of the object were the same as in the previous experiment. For viewing of the $3 \mathrm{D}$ video clip, a JVC 3D LC monitor (46-inch, polarized) was positioned at a distance of $171.9 \mathrm{~cm}$ from the subject.

The distribution of the location of the virtual image was also compared with that of convergence focal length and the focal length of lens accommodation in accordance with the chi-square test. In this study, the significant level was set to be 0.05 .

\section{RESULtS}

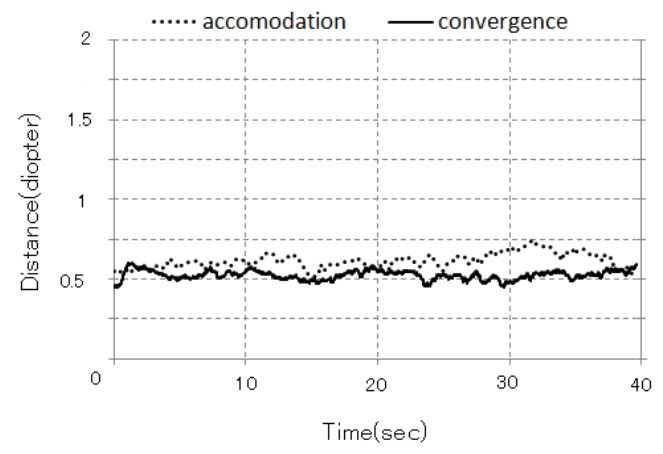

a)

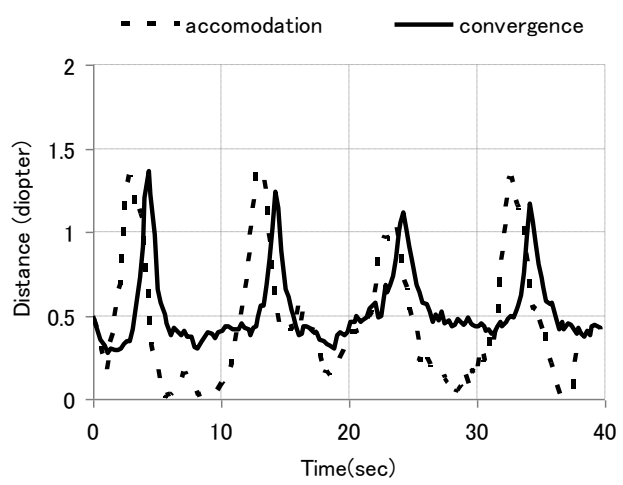

b)

Fig. 1. Typical time courses of the location of the virtual image, accommodation, and convergence focal length while viewing a) a $2 \mathrm{D}$ video clip, b) a 3D video clip.

In this study, we simultaneously measured the accommodation and convergence of subjects while they were gazing at $2 \mathrm{D}$ and $3 \mathrm{D}$ video clips in binocular vision. The results of these measurements were comparable for all subjects.

The results of this experiment are shown by a typical example while viewing $2 \mathrm{D}$ and $3 \mathrm{D}$ video clips (Fig. 1). In both these figures, "accommodation" stands for focal length of lens accommodation while "convergence" stands for convergence focal length, and "spherical object" stands for the location of the virtual image. Fig. 1 b) shows that the together and were always in agreement. The change in the diopter value occurred within a cycle of about ten seconds. Moreover, the value nearly agreed with the distance from the subject to the object (Fig. 1 b) ). In contrast, when viewing the $2 \mathrm{D}$ images, the diopter values for both accommodation and convergence were almost constant at around $0.5-0.6 \mathrm{D}$ (Fig. 1 a) ).

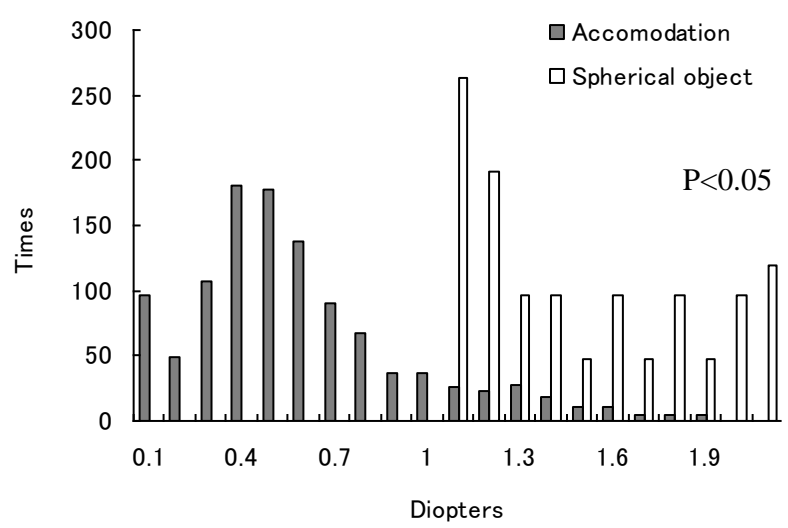

Fig. 2. Distributions of the location of the virtual image and the accommodation focal length obtained from all subjects.

The distribution of the location of the virtual image was herein compared with that of the focal length (Fig. 2). By the chi-square test, it was shown that the distribution of the location of the virtual image was significantly different from the distribution of the accommodation focal length of lens $(p<0.05)$.

\section{DISCUSSION AND CONCLUSION}

In this research, it was possible to simultaneously measure both accommodation and convergence when subjects gazed at $2 \mathrm{D}$ and $3 \mathrm{D}$ video clips (Fig. 1). In this experiment, both focal lengths were fixed at the position of the display when viewing the $2 \mathrm{D}$ images (Fig. 1 a) ). As shown in Fig. 1b, the convergence almost AGREED with the distance between a subject and a 3D image. Thus, we found that this method succeeded in measuring convergence. The distance of the accommodation focal length shifts to slight larger than that of the virtual spherical object (Fig. 2).

We used the WAM-5500 and the EMR-9 instruments. An experiment was conducted with the WAM-5500 to determine its accuracy, which was found to be $-0.01 \mathrm{D} \pm 0.38 \mathrm{D}$. That experiment also revealed that the WAM-5500 could take measurements within the range of -6.38 to +4.88D [8]. Eyestrain and transient myopia were also investigated using the WAM-5500 [9], [10]. The eye mark recorder has been used in various types of research. For example, Egami et al. [11] investigated differences according to age, tiredness and the learning effect, by showing several kinds of pictures. Sasaki [12] tried forecasting people's movements from glance data obtained from the eye mark recorder, and improved operation of a support robot based on this usage. As mentioned above, many studies have investigated the performance and characteristics of these instruments, and 
have conducted experiments using them.

We had measured the accommodation and convergence distance while subjects watched an object [13]. We calculated the convergence distance based on coordinated data for both eyes from the pupil distance. Our results showed that the accommodation and convergence of subjects changed to a position between a near and far distance as they gazed at the object. Moreover, these changes occurred at a constant frequency, the same as that of the movement of the object. Therefore, subjects viewed the object with binocular vision, and we were able to measure the results. Though these distances were nearly consistent with the distance from the subject to the object, they often were located a little beyond the object. This is thought to originate from the fact that the index is seen even when the focus is not accurate because of the depth of field. With regards to this, our study appears nearly in agreement with our previous findings [7], [14], which indicate that the lens may not be completely accommodated at about $0.4 \mathrm{D}$.

According to our previous study, accommodation does not agree strictly with the visual object but does agree with and the distance gap behind [7], [14]. On the other hand, in this study accommodation of 3D objects shifted significantly behind the $3 \mathrm{D}$ object, although its value changed with the movement of the 3D video clip (Fig. 1 b) ). As described by Hoffman et al. and Ukai et al. [5], [6], if there is inconsistency between accommodation and convergence, then accommodation of the $3 \mathrm{D}$ object would be fixed at the position of the display. However, this does not occur. Our previous study showed that the gap of accommodation behind the object was within $0.4 \mathrm{D}$, but the gap in this experiment was larger and more significant (Fig. 2).

Landy et al. [15] observed that in addition to binocular vision and motion parallax, the texture of an object is an important cue in perceiving the accurate location of an object. In this EXPERIMENT, the 3D image of the spherical object was represented as a tennis ball in order to match the conditions of the $3 \mathrm{D}$ object to those of the real ones as closely as possible. Therefore, an unnatural image seemed to pop out from the display.

For the NEXT step, it is necessary to further investigate the reliability of a measurement method by increasing the number of subjects.

\section{ACKNOWLEDGEMENT}

This work was supported in part by the Ministry of Education, Culture, Sports, Science and Technology, Grant-in-Aid for Scientific Research (B) Number 24300046.

\section{REFERENCES}

[1] V. V. Krishman, D. Shirachi, and L. Stark, "Dynamic measures of vergence accommodation," Am. J. Optom. Physiol. Opt., vol. 54, pp. 470-473, 1977.

[2] K. Ukai, Y. Tanemoto, and S. Ishikawa, "Direct recording of accommodative response versus accommodative stimulus," in Advances in Diagnostic Visual Optic, G. M. Breinin and I. M. Siegel, Ed., Berlin: Springer-Verlag, 1983, pp. 61-68.

[3] A. Cho, T. Iwasaki, and K. Noro, "A study on visual characteristics binocular 3-D images," Ergonomics, vol. 39, no. 11, pp. 1285-1293, 1996.

[4] R. Sierra, F. Uchio, N. Iguchi, and H. Taki, "Improving 3D imagery with variable convergence and focus accommodation for the remote assessment of fruit quality," in Proc. SICE-ICASE International Joint Conference 2006, 2006, pp. 3553-3558.
[5] D. M. Hoffman, A. R. Girshick, K. Akeley, and M. S. Banks, "Vergence-accommodation conflicts hinder visual performance and cause visual fatigue," Journal of vision, vol. 8, no. 3, article 33, pp. 1-30, 2008.

[6] K. Ukai and P. A. Howarth, "Visual fatigue caused by viewing stereoscopic motion images: Background, theories, and observation," Displays, vol. 29, pp. 106-116, 2008.

[7] M. Miyao S. Y. Ishihara, S. Saito, T. A. Kondo, H. Sakakibara, and H Toyoshima, "Visual accommodation and subject performance during a stereographic object task using liquid crystal shutters," Ergonomics, vol. 39, no. 11, pp.1294-1309, 1996.

[8] A. L. Sheppard and L. N. Davies, "Clinical evaluation of the Grand Seiko Auto Ref/Keratometer WAM-5500," Ophthal. Physiol. Opt., vol. 30, pp. 143-151, 2010

[9] C. Tosha, E. Borsting, W. H. Ridder 3rd, and C. Chase, "Accommodation response and visual discomfort," Ophthal. Physiol. Opt., vol. 29, pp. 625-633, 2009.

[10] E. Borsting, C. Tosha, C. Chase, and W. H. Ridder 3rd, "Measuring near-induced transient myopia in college students with visual discomfort," American Academy of Optometry, vol. 87, no. 10, pp. 760-766, 2010.

[11] C. Egami, K. Morita, T. Ohya, Y. Ishii, Y. Yamashita, and T. Matsuishi, "Developmental characteristics of visual cognitive function during childhood according to exploratory eye movements," Brain \& Development, vol. 31, pp. 750-757, 2009.

[12] T. Sakaki, "Estimation of intention of user arm motion for the proactive motion of upper extremity supporting robot," in Proc. 2009 IEEE 11th International Conference on Rehabilitation Robotics, Kyoto, 2009, pp. 486-490.

[13] T. Shiomi, H. Hori, K. Uemoto, M. Miyao, H. Takada, S. Hasegawa, M. Omori, T. Watanabe, and H. Ishio, "Comparison of simultaneous measurement of lens accommodation and convergence in natural vision and 3D vision," in Proc. Society for Information Display 2012, Boston, 2012, pp. 32.

[14] Y. Otake, M. Miyao, S. Ishihara, M. Kashiwamata, T. Kondo, H. Sakakibara, and S. Yamada, "An experimental study on the objective measurement of accommodative amplitude under binocular and natural viewing conditions," Tohoku J. Exp. Med., vol. 170, pp. 93-102, 1993.

[15] M. S. Landy, L. T. Maloney, E. B. Johnston, and M. Young, "Measurement and modeling of depth cue combination: in defense of weak fusion," Vision Research, vol. 35, no. 3, pp. 389-412, 1995.

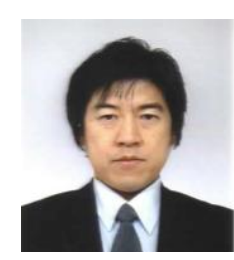

Takehito Kojima was born at Nagoya, Japan. The author received M.Phil. from Nagoya Institute of Technology in 2012. The author's research lies in Human-Computer Interaction (HCI). More specially, the author enjoys doing research in ergonomics for 3-D display technology and mobile display devices.

$\mathrm{He}$ is a student of the doctoral course at Graduate School of Information Science, Nagoya University. His research is broadly included in the ergonomics and he is mainly researching stereoscopic vision from a point of view of human visual functions.

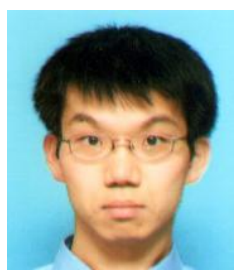

Yasuyuki Matsuura was born at Nagoya, Japan. The author received $\mathrm{PhD}$ in science from Nagoya City University (Nagoya, Japan) in 2009. During 2009-2010, he worked Researcher in Department of Hygiene, Graduate School of Medicine, Nagoya City University. During 2010-2011, he worked for Nagoya University as a postdoctoral research fellow.

$\mathrm{He}$ is currently a postdoctoral research fellow at Headquarters for Innovative Society-Academia Cooperation, University of Fukui, Japan since 2011. His research lies in Medical Engineering (especially, Biological Signal Processing). He is currently focusing his studies on computational modeling of electrogastrogram, and on application of non-linear analysis methods to characterize biological signals (Electrocardiogram, Electrogastrogram, Stabilogram, et al.).

$\mathrm{He}$ is a member of IEEE and the Engineering in Medicine and Biology.

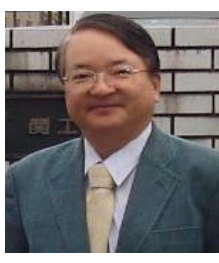

Masaru Miyao was born at Tokyo, Japan. The author received MD from Nagoya University in 1977 and $\mathrm{PhD}$ in medicine from Nagoya University in 1982. Broadly, the author's research lies in Human-Computer Interaction (HCI). More specially, the author enjoys doing research in ergonomics for 3-D display technology and mobile interaction including Head Mounted Displays (HMDs). The author's research is focused on building and evaluating systems designed to 
human vision, especially accommodation and convergence for stereoscopic displays, and presently studying how to make comfortable 3-D displays and 3-D movie contents. He is currently a professor at the Graduate School of Information Science, Nagoya University since 2002.

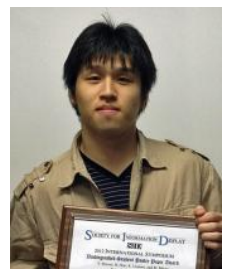

Tomoki Shiomi was born in March, 1986. He obtained his bachelor of science in 2008 and master of science in 2010 from Ritsumeikan University. And he obtained $\mathrm{PhD}$ in 2013 from Nagoya University.

He was a student of the doctoral course at Graduate school of Information Science, Nagoya University till March 2013. His research is broadly included in the ergonomics and he is mainly researching stereoscopic vision from a point of view of human visual functions. He moved to a labor standard orthority since April 2013.

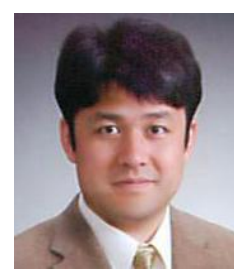

Hiroki Takada was born in July 1973 at Kuwana, Japan. In 2002, he graduated from Graduate School of Mathematics, Nagoya University where he completed his Under-graduate and Post-graduate study. He received doctorate by way of dissertation (DSc) in 2004. During 2002-2006, he worked for Nagoya University as a postdoctoral research fellow in several fields such as physics, environmental medicine, and computational science. Since 2006, he became associate professor in Gifu. He worked for Department of Radiology, Gifu University of Medical Science. In the recent personal changes, he transferred to Department of Human \& Artificial Intelligent Systems, University of Fukui, and also, a guest researcher for Aichi Medical University School of Medicine. Broadly, his research lies in Mathematical Physics (especially, Stochastic Process Theory). Applying this theoretical background, the author enjoys doing research in Environmental Physiology for preventive medicine, biosignal such as electrocardiogram, continuous blood pressure, cerebral blood flow, micro-neurogram, stabilogram, electromyogram, skin potential, and electrogastrogram. The author received many awards including an award for encouragement from Society for Science on Form in 2002. He serves as an executive editor of "Journal of Sports Medicine and Doping Study (USA)". He is also an associate editor in chief of "FORMA", a member of IEEE and Program Committee of "International Conference on Bio-inspired systems and signal processing". 